Chronic Obstructive Pulmonary Diseases: Journal of the COPD Foundation

\author{
Original Research
}

\title{
Benefits Among Patients with Alpha-1 Antitrypsin Deficiency Enrolled in a Disease Management and Prevention Program
}

\author{
Jordan T. Perkins, $\mathrm{MPH}^{1}$ Radmila Choate, $\mathrm{MPH}^{1}$ David M. Mannino, MD ${ }^{1,2}$ Steven R. Browning, PhD, $\mathrm{MSPH}^{2}$ \\ Robert A. Sandhaus, $\mathrm{MD}, \mathrm{PhD}^{3}$
}

\section{Abstract}

Rationale: Alpha-1 antitrypsin deficiency (AATD) is characterized by decreased circulating levels or activity of the serum protein, alpha-1 antitrypsin, which increases risk for chronic lung or liver injury and may lead to diseases such as chronic obstructive pulmonary disease (COPD). Currently there is no cure for AATD, and it is largely controlled through disease management and augmentation therapy. This study was designed to describe characteristics of patients enrolled in a disease management and prevention program.

Methods: Data from questionnaires administered by AlphaNet were obtained on 4747 AATD patients and included demographic information, medical history, lifestyle choices, and adherence to the Alpha-1 Disease Management and Prevention Program (ADMAPP). A total of 1221 participants (25.72\%) had missing adherence information and were excluded, leaving a final study population of 3526. Questionnaire answer dates ranged from May 29, 2008 to February 14, 2015. Logistic regression was used to adjust for demographic factors and comorbidities, comparing the populations stratified by adherence to ADMAPP.

Results: After adjustment for age, sex, race, Charlson Comorbidity Index, and income level, individuals who selfreported any adherence to ADMAPP were more likely to feel informed about their condition (odds ratio[OR] adj 4.95, 95\% confidence interval[CI][3.24, 7.57]), and be taking preventive measures, such as smoking cessation (OR adj $0.47,95 \%$ CI $[0.31,0.70])$, appropriate immunizations, and self-reported exercise (ORadj $2.07,95 \%$ CI [1.74, 2.47]).

Conclusions: This study suggests that ADMAPP may be a useful tool for informing and improving preventive measures taken by individuals with AATD. Future studies are needed to clarify the observed associations and study additional outcomes.

\footnotetext{
Abbreviations: alpha-1 antitrypsin deficiency, AATD; chronic obstructive pulmonary disease, COPD; alpha- 1 disease management and prevention program, ADMAPP; odds ratio, OR; confidence interval, CI; alpha-1 antitrypsin, AAT; Big Fat Reference Guide to Alpha-1, BFRG; body mass index, BMI; Charlson Comorbidity Index, CCI

Funding Support: This study was funded by an unrestricted research grant from AlphaNet.

Date of Acceptance: October 19, 2016

Citation: Perkins JT, Choate R, Mannino DM, Browning SR, Sandhaus RA. Benefits among patients with alpha- 1 antitrypsin deficiency enrolled in a disease management and prevention program. Chronic Obstr Pulm Dis. 2017;4(1):56-64. doi: https://doi.org/10.15326/ jcopdf.4.1.2016.0161
}

\footnotetext{
1 Department of Preventive Medicine and Environmental Health, University of Kentucky College of Public Health, Lexington

2 Department of Epidemiology, University of Kentucky College of Public Health, Lexington

3 Division of Pulmonary, Critical Care and Sleep Medicine, National Jewish Health, Denver, Colorado
} 


\section{Address correspondence to:}

David M. Mannino, MD

Department of Preventive Medicine and Environmental Health University of Kentucky College of Public Health

111 Washington Avenue

Lexington, KY 40536

Phone: 8592182099

E-mail:dmannino@uky.edu

\section{Keywords:}

Alpha-1 antitrypsin deficiency; AATD; chronic obstructive pulmonary disease; COPD

\section{Introduction}

Alpha-1 antitrypsin deficiency (AATD) was first described in 1963 by Carl-Bertil Laurell and Sten Eriksson who observed that low levels of the alpha-1 antitrypsin (AAT) protein were associated with precocious emphysema. ${ }^{1}$ This discovery, coupled with the association of AATD with childhood and adult liver disease has led to the condition being recognized as a predisposing factor for the development of liver disease, emphysema, certain vasculitides, as well as chronic obstructive pulmonary disease (COPD), in general. ${ }^{2}$ While the condition has been identified for over 50 years, knowledge about prevention and treatment of the disease and its prevalence is somewhat limited.

AATD is a heritable condition that, as its name suggest, results in an individual having lower circulating levels of the AAT protein. Lower levels of this protein have been linked to a number of diseases; the majority of which are related to impaired lung function and liver disease. ${ }^{3}$ The most common forms of AATD are the result of single base substitutions that lead to a change in the amino acid composition of the protein. ${ }^{2}$ More than 200 of these mutations have been identified in the SERPINA 1 gene, which contains the genetic information for the AAT protein. The effects of these mutations can range from harmless to decreases in activity or translational errors that create a protein that is ultimately degraded. ${ }^{2,4}$

Normally, the AAT protein functions as a serine protease inhibitor, with the most notable target being neutrophil elastase. When neutrophils are recruited to sites of inflammation, elastase is released to combat the source of inflammation, but due to its broad proteolytic activity, also can attack host proteins. ${ }^{2}$ AAT helps to balance the protective effects of the elastase, while limiting damage to native tissues. Therefore, a lack of activity or decrease in AAT level results in uncontrolled enzymatic elastase activity and increased host damage following inflammation. The majority of disease associated with AATD is associated with the pulmonary system, such as emphysema and bronchiectasis, with a smaller proportion of patients having hepatic and vasculitic manifestations. ${ }^{2,3,5,6}$

Currently there are no cures for AATD, but several treatment options are available, most of which are the same used to treat COPD. ${ }^{5}$ Augmentation therapy, using purified human plasma-derived AAT protein administered intravenously on a weekly basis, is the only specific therapy currently available and is reserved for those with documented pulmonary emphysema. Additional therapies are in development, such as gene-based therapeutics. ${ }^{5,7}$ The efficacy of augmentation therapy has been evaluated in a number of smaller clinical trials ${ }^{8-10}$ as well as a recent wellpowered randomized, blinded, placebo-controlled trial demonstrating a reduction in the loss of lung tissue among patients on augmentation therapy. ${ }^{11}$

To help patients, families of patients, and health care providers navigate the wealth of information on AATD, AlphaNet, a not-for-profit organization founded in 1995, has developed the Alpha-1 Disease Management and Prevention Program (ADMAPP). The program provides support as well as education on the disease. A large part of the educational component is devoted to prophylactic measures that individuals with AATD can take to help decrease risk of future disease complications. Specifically, preventive measures, such as smoking cessation, nutrition and exercise improvement, rapid recognition and treatment of exacerbations, identification and treatment of comorbid conditions, and immunization against influenza virus, hepatitis, and pneumonia, are emphasized in ADMAPP. ADMAPP encompasses many factors including education and interventions with the goal of optimizing clinical and economic outcomes for persons with AATD. ${ }^{12}$ The program provides a variety of resources, including a unique telemonitoring system that enables persons with AATD to interact with coordinators (who also have AATD) on a monthly basis. The cornerstone of ADMAPP is the Big Fat Reference Guide to Alpha-1(BFRG), which is a free resource provided by AlphaNet. ${ }^{12}$ It is a comprehensive guide meant for patients with AATD, their families, and medical providers and is one of the main components of the ADMAPP. During the first 6 months after entry into ADMAPP, coordinators interact with the patient to review the various components of self-management as described in the BFRG and document each participant's 
understanding of the material. A large part of adherence to ADMAPP is reading and complying with the suggestions in the BFRG, but this act alone does not necessarily signify adherence, as the BFRG is one part of ADMAPP. This study was designed to assess associations between self-reported adherence to the AlphaNet ADMAPP and the prophylactic measures that are encouraged by the program as well as determining if any of these associations are a result of demographic and health differences between individuals who are ADMAPP adherent and those who are not.

\section{Methods}

\section{Study Design and Population}

This study uses data gathered from questionnaires that individuals with AATD in the AlphaNet system completed, which have been described elsewhere. ${ }^{13}$ The questionnaires are administered via telephone by trained AlphaNet coordinators who are assigned to participants based on geographical proximity, and take roughly an hour to complete. The dataset is a sample of 4747 individuals enrolled in ADMAPP, containing qualitative, self-reported, questionnaire responses, with answer dates ranging from May 29, 2008 to February 14,2015 . It is important to note that all participants in the AlphaNet program evaluated in this study entered the program because they are currently receiving or are about to begin intravenous augmentation therapy for lung disease due to AATD.

The study population was determined by the response to 2 questions regarding adherence to ADMAPP. Participants were asked if they currently followed the guidelines of ADMAPP and for their estimated percentage of adherence with the program. Thus, the study definition of adherence was defined as any adherence to ADMAPP versus no adherence to ADMAPP. Of the 4747 participants, 1605 (33.8\%) reported any adherence, 1921 (40.5\%) reported no adherence, and 1221 (25.7\%) had missing adherence information, leaving a final study population of 3526 (Figure 1).

Lifestyle choices (e.g., smoking, drinking, exercise habits, immunizations), health status (e.g., body mass index [BMI], perceived health) and knowledge of the disease were compared between the adherent and non-adherent groups. Exercise was originally reported in terms of frequency of the activity as well as the location of the exercise. This information was combined so that any indication of exercise was considered a positive response for exercise. For this study, socio-demographic characteristics (sex, age, race, marital status, income, BMI, smoking status) as well as current medical conditions and information specific to AATD and ADMAPP, were also considered for possible confounding.

\section{Charlson Comorbidity Index}

To ensure differences between the 2 groups were not due to unequal distribution of medical conditions between the 2 stratified populations, the Charlson Comorbidity Index (CCI) was used. Briefly, the CCI uses categories of comorbidities that are assigned a weighted integer value based on the risk of mortality. Mortality risk increases proportionally with the CCI score. ${ }^{14}$ Missing or negative answers for each condition were given a score of zero.

\section{Statistical Methods}

Frequencies and percentages were reported for categorical variables for the entire population as well as

\section{Figure 1. Flowchart of the Study Population}

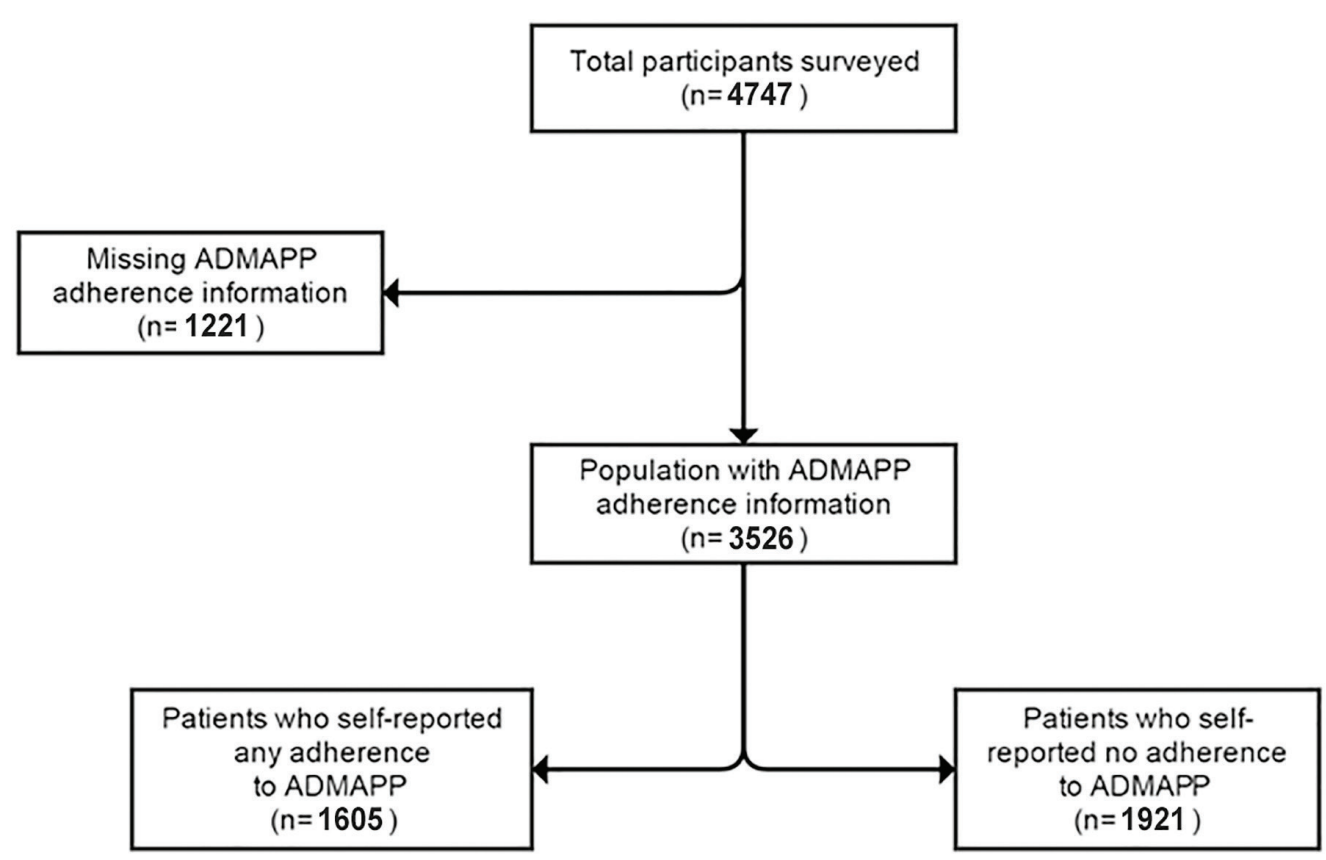


the subgroups within the population and were compared using chi-square tests. Continuous variables were reported as means and standards deviations and were compared using t-tests. $P$ values were reported for both continuous and categorical variables, with significance threshold being a p value less than 0.05 .

Crude odds ratios (OR) and the respective 95\% confidence intervals (CI) were calculated for all outcomes of interest, comparing the 2 stratified populations. Adjusted odds ratios were also calculated for the outcomes of interest, controlling for age, sex, CCI values, and income. The covariates used in adjustment were chosen based on differences in population demographics as well as potential confounding. The covariates were then considered for model inclusion using a stepwise technique described elsewhere. ${ }^{15}$ All statistical analyses were performed using SAS software, Version 9.3 of the SAS System for Windows.

\section{Results}

Overall, the sample ( $\mathrm{n}=3526)$ was fairly evenly split between genders (53\% male, 46\% female, 1\% missing), with no statistical differences in age $(p=0.117)$, CCI score ( $p=0.760$ for continuous classification and $p=0.927$ for categorical classification), or gender $(p=0.059)$ between the stratified groups (any versus no adherence to ADMAPP). Significant differences were observed in marital status $(p=0.004)$, income $(p=<0.001)$, and race $(p=<0.001)$, although racial differences were not considered significant as the majority of the sample was white (Table 1).

Adherent ADMAPP users had statistically significant increases in the proportion of individuals who selfreported having read any part of the BFRG, a collection of free educational material on AATD (Table 2). After adjustment, adherent individuals had significantly higher odds of reading the BFRG (OR adj 6.44, 95\% CI $[5.45,7.62])$ as well as increased odds of feeling comfortable with their disease knowledge ( $O R_{\text {adj }} 4.95$, 95\% CI [3.24, 7.57]) (Table 3).

Initial analysis yielded statistically significant differences in the distribution of the results for perception of weight, fitness, and overall health and in increase in self-reported exercise (Table 2). Following logistic regression adjustment, adherent users had higher odds of reporting a fitness level better than "out of shape" (ORadj 1.66, 95\% CI [1.43, 1.94]) and exercise, regardless of regularity or location (OR adj $2.07,95 \% \mathrm{CI}$ $[1.74,2.47])$. Comparisons between BMI classifications as well as perceived weight and health were not statistically significant prior to, or after adjustment (Table 3).

Adherent ADMAPP users were also more likely to report immunizations for all of the diseases examined (Table 4). Logistic regression generated increased odds for influenza (OR $\left.\mathrm{R}_{\text {adj }} 1.34,95 \% \mathrm{CI}[1.08,1.68]\right)$, Hepatitis A [(OR adj 1.41, 95\% CI $[1.20,1.66])$, and Hepatitis B (ORadj 1.62 95\% CI [1.37, 1.91]) immunizations. Odds of being current smokers were also half in the adherent population (OR $\mathrm{Odj}_{\mathrm{j}} 0.47$, 95\% CI [0.31, 0.70]) (Table 3). Interestingly, ADMAPP adherent users were more likely to report drinking alcohol, but did not drink in excess, compared to the non-adherent population (Table 4). This difference in drinking habits was found to be statistically insignificant following adjustment (Table 3).

\section{Discussion}

Self-reported comfort with knowledge of AATD, as well as the use of the BFRG, were associated with the largest odds ratios of all of the outcomes examined in this study (Table 4). The information contained within the BFRG is extensive, covering a broad range of topics related to AATD management and disease prevention. The guide and the educational contents are the largest part of the ADMAPP program, so these results suggest that individuals in the program are reading the guide, which may correlate with increased comfort with their disease knowledge and translate into prophylactic behavioral changes. These results must be interpreted cautiously though, as this is not a validated measure of change in participant knowledge and is relative to the person answering the question. It does not measure whether or not the knowledge is correct, merely whether or not they feel comfortable with their knowledge. A number of questions included in the analysis dealt with an individual's perception of his/her general health and fitness as well as his/her exercise habits. Initial comparisons suggested several instances of distinction between the 2 samples (Table 2).

The answers to all of the perception questions are subjective. One person's perception of "very fit," for example, may not match another person's perception. Furthermore, exercise habits were originally categorized based on regularity and location of the exercise. These answers were combined so that any indication of exercise was considered a positive response for exercise. 


\section{Table 1. Characteristics of Total and Stratified Population}

\begin{tabular}{|c|c|c|c|c|}
\hline & $\begin{array}{l}\text { Final Sample } \\
\qquad(n=3526)^{a}\end{array}$ & $\begin{array}{c}\text { No ADMAPP } \\
\text { Adherence } \\
(n=1921)\end{array}$ & $\begin{array}{l}\text { Any ADMAPP } \\
\text { Adherence } \\
(n=1605)\end{array}$ & $p$-value \\
\hline Age (Mean $\pm \mathrm{SD})$ & $56.62 \pm 11.76$ & $56.34 \pm 12.19$ & $56.96 \pm 11.22$ & 0.117 \\
\hline Sex & & & & 0.059 \\
\hline Male & $1832(52.57)$ & $1026(53.41)$ & 806 (50.22) & \\
\hline Female & $1653(46.43)$ & $873(45.45)$ & $780(48.60)$ & \\
\hline Marital Status & & & & 0.004 \\
\hline Married & $2183(61.91)$ & $1141(59.40)$ & $1042(64.92)$ & \\
\hline Single & $1246(35.34)$ & $715(37.22)$ & $531(33.08)$ & \\
\hline Race & & & & $<0.001$ \\
\hline White & 3388 (96.06) & $1825(95.00)$ & $1563(97.38)$ & \\
\hline Non-White & $85(2.41)$ & $61(3.18)$ & $24(1.50)$ & \\
\hline Income & & & & $<0.001$ \\
\hline$<\$ 20,000$ & $935(26.52)$ & $564(35.97)$ & $371(28.06)$ & \\
\hline$\$ 20,000-\$ 40,000$ & 775 (21.98) & $414(26.40)$ & $361(27.31)$ & \\
\hline$\$ 40,000-\$ 60,000$ & $501(14.21)$ & 267 (17.03) & $234(17.70)$ & \\
\hline$\$ 60,000-\$ 80,000$ & $294(8.34)$ & $147(9.38)$ & $147(11.12)$ & \\
\hline$\$ 80,000-\$ 100,000$ & $159(4.51)$ & $75(4.78)$ & $84(6.35)$ & \\
\hline$>\$ 100,000$ & $226(6.41)$ & $101(6.44)$ & $125(9.46)$ & \\
\hline CCI (Mean \pm SD) & $0.77 \pm 1.28$ & $0.78 \pm 1.29$ & $0.76 \pm 1.27$ & $0.760^{\mathrm{b}}$ \\
\hline 0 & $2217(62.88)$ & $1200(62.47)$ & 1017 (63.36) & $0.927^{\mathrm{c}}$ \\
\hline 1 & $548(15.54)$ & 308 (16.03) & $240(14.95)$ & \\
\hline 2 & $427(12.11)$ & $231(12.02)$ & $196(12.21)$ & \\
\hline 3 & $159(4.51)$ & $84(4.37)$ & $75(4.67)$ & \\
\hline 4 & $84(2.38)$ & $48(2.50)$ & $36(2.24)$ & \\
\hline 5 & $40(1.13)$ & $20(1.04)$ & $20(1.25)$ & \\
\hline$>6$ & $51(1.45)$ & 30 (1.56) & $21(1.31)$ & \\
\hline
\end{tabular}

Note: All statistics are reported as frequency (percentage) unless otherwise indicated. Comparisons were made between the 2 stratified populations. Percentages may not add up to $100 \%$ due to missing information.

Bolded $p$-values are statistically significant.

a 1221 individuals with missing adherence data were excluded; the final sample figures were not used in the comparison calculations

${ }^{b}$ Test statistics for t-test, comparing continuous classification of CCI

${ }^{\mathrm{c}}$ Test statistics for chi-squared, comparing categorical classification of CCI

ADMAPP=alpha-1 disease management and prevention program; CCI=Charlson Comorbidity Index

For the purposes of this study, the effort of exercise was more important than the location or regularity of the exercise.

Even with these limitations, the conclusion that ADMAPP-adherent individuals were more likely to exercise and have a positive image of their fitness remains strong. Considering the lack of temporality of this data, it is impossible to state how long people had been enrolled and therefore, the impact of the program may just not be as evident. This may explain the lack of significant changes in weight, as these would have been the result of multiple aspects of an individual's lifestyle over a sustained amount of time.

The lack of statistically significant results for perceived health comparisons may have also been affected by how long participants had been in the 


\section{Table 2. Comparison of Disease Knowledge and Health/Health Perception Between Patients With Any and No ADMAPP Adherence}

\begin{tabular}{|c|c|c|c|}
\hline & $\begin{array}{l}\text { No ADMAPP } \\
\text { Adherence } \\
(n=1921)\end{array}$ & $\begin{array}{c}\text { Any ADMAPP } \\
\text { Adherence } \\
(n=1605)\end{array}$ & p-value \\
\hline \multicolumn{3}{|c|}{ Comfort with Disease Knowledge } & $<0.001$ \\
\hline Not comfortable & $147(7.65)$ & $36(2.24)$ & \\
\hline Somewhat comfortable & $960(49.97)$ & $574(35.76)$ & \\
\hline Comfortable & $782(40.71)$ & 974 (60.69) & \\
\hline \multicolumn{3}{|l|}{ Read Any Part of BFRG } & $<0.001$ \\
\hline Yes & $518(26.97)$ & $1126(70.16)$ & \\
\hline No & $1361(70.85)$ & $461(28.72)$ & \\
\hline \multicolumn{3}{|l|}{ Body Mass Index } & 0.274 \\
\hline Underweight & $91(4.74)$ & $76(4.74)$ & \\
\hline Normal & $655(34.10)$ & $580(36.14)$ & \\
\hline Overweight & $515(26.81)$ & 439 (27.35) & \\
\hline Obese & $443(23.06)$ & $328(20.44)$ & \\
\hline \multicolumn{3}{|l|}{ Perceived Weight } & 0.084 \\
\hline Underweight & $225(11.71)$ & $184(11.46)$ & \\
\hline About right & $716(37.27)$ & $665(41.43)$ & \\
\hline Overweight & $930(48.41)$ & $736(45.86)$ & \\
\hline \multicolumn{3}{|l|}{ Perceived Fitness } & $<0.001$ \\
\hline Out of shape & $982(51.12)$ & $632(39.38)$ & \\
\hline Pretty fit & $502(26.13)$ & $531(33.08)$ & \\
\hline Getting fit & $323(16.81)$ & $351(21.87)$ & \\
\hline Very fit & $45(2.34)$ & $61(3.80)$ & \\
\hline \multicolumn{3}{|l|}{ Perceived Health } & $<0.001$ \\
\hline Poor & 378 (19.68) & $273(17.01)$ & \\
\hline Fair & $826(43.00)$ & $646(40.25)$ & \\
\hline Good & 601 (31.29) & 589 (36.70) & \\
\hline Excellent & 58 (3.02) & 74 (4.61) & \\
\hline \multicolumn{3}{|l|}{ Exercise Habits } & $<0.001$ \\
\hline Exercise & 1103 (57.42) & $1192(74.27)$ & \\
\hline Don't exercise & $661(34.41)$ & $323(20.12)$ & \\
\hline
\end{tabular}

Note: Note: All statistics are reported as frequency (percentage) unless otherwise indicated Bolded $p$-values are statistically significant.

ADMAPP=alpha- 1 disease management and prevention program; $B F R G=B i g$ Fat Reference Guide to Alpha-1

program as well as a lack of a clear definition of what is meant by the word. Health is an encompassing term and can mean different things to different people. Without explicitly stating what aspects of health the question is referring to (e.g., mental health, physical health), any results from this question would be difficult to interpret in the proper context.

The results regarding the prophylactic measures taken by adherent individuals are significant. Since there is currently no cure for AATD, much of the treatment information for individuals with the condition focuses on preventive measures that can be taken to decrease the burden of the disease on the individual. Generally, these measures are focused on preventing xenobiotic and infectious insults to the lungs or liver, as well as healthy lifestyle choices. Ultimately, the aim is to avoid a proinflammatory environment.

One of the largest potential contributors to inflammation in the lungs is smoking, which can also increase host damage due to oxidative inactivated AAT protein, and as such, is a major focus of ADMAPP. This study found that ADMAPP-adherent individuals had half the odds of being active smokers (Table 3 ). In addition, these individuals were more likely to be immunized against influenza as well as Hepatitis A and B, which can be more serious in individuals with AATD, and are recommended immunizations in the BFRG. Taken together, these results are encouraging and suggest that ADMAPP-adherent individuals have higher odds of taking steps to reduce the chances of aggravating their condition.

There are several important limitations of this study that should be considered along with the results. First, all of the information, including the medical history, was collected via a self-reported questionnaire which brings into question the validity of the information provided. The self-reported nature of the study brings in recall bias (how accurate a person was able to remember information), reporting bias (selective revealing or suppressing of information by the participant), as well as a degree of social desirability bias (participants providing answers that they think 


\section{Table 3. Crude and Adjustment Odds Ratios, Comparing Patients With Any and No ADMAPP Adherence}

\begin{tabular}{|c|c|c|}
\hline & $\begin{array}{c}\text { Crude OR } \\
\text { (95\% CI) }\end{array}$ & $\begin{array}{c}\text { Adjusted OR } \\
(95 \% \mathrm{Cl})\end{array}$ \\
\hline Comfort with Disease Knowledge (comfortable vs. not comfortable) & 5.09 (3.49-7.41) & 4.95 (3.24-7.57) \\
\hline Have Read Any Part of BFRG (yes vs. no) & $6.42(5.53-7.44)$ & $6.44(5.45-7.62)$ \\
\hline \multicolumn{3}{|l|}{ Body Mass Index } \\
\hline (normal vs. overweight/obese) & $1.11(0.96-1.28)$ & $1.04(0.88-1.23)$ \\
\hline (normal vs. underweight) & $1.06(0.77-1.47)$ & $0.99(0.68-1.44)$ \\
\hline Perceived Fitness (pretty fit/getting fit/very fit vs. out of shape) & $1.68(1.47-1.93)$ & $1.66(1.43-1.94)$ \\
\hline \multicolumn{3}{|l|}{ Perceived Weight } \\
\hline (about right vs. underweight) & $1.14(0.91-1.42)$ & $1.03(0.80-1.33)$ \\
\hline (about right vs. overweight) & $1.17(1.02-1.35)$ & $1.12(0.95-1.31)$ \\
\hline Perceived Health (good/excellent vs. poor/fair) & $1.32(1.15-1.51)$ & $1.16(0.98-1.36)$ \\
\hline Exercise (yes vs. no) & $2.21(1.89-2.59)$ & $2.07(1.74-2.47)$ \\
\hline Still Smoking (yes vs. no) & $0.40(0.27-0.58)$ & $0.47(0.31-0.70)$ \\
\hline Drinker (yes vs. no) & $1.25(1.09-1.43)$ & $1.12(0.96-1.31)$ \\
\hline \multicolumn{3}{|l|}{ Vaccinations } \\
\hline Pneumonia in last 6 years (yes vs. no) & $1.29(1.05-1.58)$ & $1.14(0.90-1.44)$ \\
\hline Flu vaccine in last year (yes vs. no) & $1.43(1.17-1.75)$ & $1.34(1.08-1.68)$ \\
\hline Hepatitis A (yes vs. no) & $1.51(1.30-1.74)$ & $1.41(1.20-1.66)$ \\
\hline Hepatitis B (yes vs. no) & $1.74(1.50-2.02)$ & $1.62(1.37-1.91)$ \\
\hline
\end{tabular}

a Adjusted for age, sex, race, CCI score, and income level

Bolded $p$-values are statistically significant.

$\mathrm{ADMAPP}=$ alpha- 1 disease management and prevention program; $\mathrm{OR}=$ odds ratio; confidence interval $=\mathrm{CI} ; \mathrm{BFRG}=\mathrm{Big} \mathrm{Fat}$ Reference Guide for Alpha-1; CCI=Charlson Comorbidity Index

are correct or desired). Participants were also asked if they had ever been immunized against Hepatitis A and $B$ therefore, these immunizations may have been completed prior to their enrollment in ADMAPP. The data also lacked a longitudinal component, meaning the associations presented cannot be used to infer causality. Missing entries were also a limitation, with $25.72 \%$ of participants providing no information for the question concerning ADMAPP adherence, meaning our results may under- or overestimate the true results.

While missing observations continue to be a limitation of this study, the large sample size and demographics for those missing adherence data helps to alleviate some of the concerns about the missing information. As this study indicates an association between ADMAPP adherence and several preventive outcomes, future studies need to be conducted using longitudinal data on this population to determine if this relationship is causal. Ultimately, the results of this study display the positive impact ADMAPP has on individuals with AATD. Individuals who self-reported any adherence to the program were more likely to be informed about their condition and take preventive measures, such as smoking cessation, and getting immunized against conditions that could magnify the effects of AATD, while also self-reporting they exercise.

\section{Acknowledgements}

The authors wish to thank the investigators, patients, and coordinators of AlphaNet for access to their data.

\section{Declaration of Interest}

DM has received honoraria/consulting fees and served on speaker bureaus for GlaxoSmithKline PLC, Novartis Pharmaceuticals, Pfizer Inc., Boehringer-Ingelheim, 


\section{Table 4. Comparing Exacerbating and Prophylactic Factors Taken by Patients With Any and No ADMAPP Adherence}

\begin{tabular}{|c|c|c|c|}
\hline & $\begin{array}{l}\text { No ADMAPP } \\
\text { Adherence } \\
(n=1921)\end{array}$ & $\begin{array}{c}\text { Any ADMAPP } \\
\text { Adherence } \\
(n=1605)\end{array}$ & p-value \\
\hline \multicolumn{3}{|c|}{ Pneumonia Vaccine in Last 6 Years } & 0.017 \\
\hline Yes & $1586(82.56)$ & $1391(86.67)$ & \\
\hline No & 255 (13.27) & 174 (10.84) & \\
\hline \multicolumn{3}{|c|}{ Flu Vaccine in Last Year } & $<0.001$ \\
\hline Yes & $1592(82.87)$ & $1409(87.79)$ & \\
\hline No & $285(14.84)$ & $176(10.97)$ & \\
\hline \multicolumn{3}{|c|}{ Hepatitis A Vaccine, Ever } & $<0.001$ \\
\hline Yes & 677 (35.24) & 733 (45.67) & \\
\hline No & $864(44.98)$ & $620(38.63)$ & \\
\hline \multicolumn{3}{|c|}{ Hepatitis B vaccine, Ever } & $<0.001$ \\
\hline Yes & $957(49.82)$ & $1046(65.17)$ & \\
\hline No & $681(35.45)$ & $427(26.60)$ & \\
\hline \multicolumn{3}{|l|}{ Smoking Status } & 0.831 \\
\hline Never smoked & $463(24.10)$ & $398(24.80)$ & \\
\hline Have smoked & $1415(73.66)$ & $1196(74.52)$ & \\
\hline \multicolumn{3}{|l|}{ Still smoking } & $<0.001$ \\
\hline Yes & $107(5.98)$ & $38(3.18)$ & \\
\hline No & $1295(91.52)$ & $1157(96.74)$ & \\
\hline \multicolumn{3}{|l|}{ Drinking Habits } & 0.001 \\
\hline Don't drink & $1093(56.90)$ & $842(52.46)$ & \\
\hline Drink & $757(39.41)$ & $727(45.30)$ & \\
\hline $\begin{array}{l}\text { Weekly Drinks } \\
(\text { Mean } \pm \text { SD) }\end{array}$ & $5.79 \pm 7.07$ & $5.50 \pm 6.55$ & 0.416 \\
\hline
\end{tabular}

Note: All statistics are reported as frequency (percentage) unless otherwise indicated Bolded $p$-values are statistically significant.

$\mathrm{ADMAPP}=$ alpha-1 disease management and prevention program
AstraZeneca PLC, Forest Laboratories Inc., Merck, Amgen, and Sunovion. Furthermore, he has received royalties from Up-to-Date, is on the Board of Directors of the COPD Foundation, and has been compensated as a medical expert in legal cases. JTP, RC, and SRB report no conflicts of interest. RAS has received research funding from Grifols, CSL Behring, AstraZeneca, and the National Institutes of Health/National Heart Lung and Blood Institute. He has served as an advisor or consultant for Grifols, CSL Behring, Baxter, AstraZeneca, and Alnylam. $\mathrm{He}$ is on the Board of Directors of AlphaNet and a member of the Medical and Scientific Advisory Committee of the COPD Foundation and is the medical director of the Alpha-1 Foundation and AlphaNet. 


\section{References}

1. Laurell CB, Eriksson S. The electrophoretic alpha1-globulin pattern of serum in alpha1-antitrypsin deficiency. 1963. COPD. 2013;10 (Suppl 1):3-8.

doi: https://doi.org/10.3109/15412555.2013.771956

2. Stoller JK, Aboussouan LS. A review of alpha1-antitrypsin deficiency. Am J Respir Crit Care Med. 2012;185(3):246-259. doi: https://doi.org/10.1164/rccm.201108-1428CI

3. Needham M, Stockley RA. Alpha 1-antitrypsin deficiency. 3: Clinical manifestations and natural history. Thorax. 2004; 59(5):441-445. doi: https://doi.org/10.1136/thx.2003.006510

4. Fregonese L, Stolk J, Frants RR, Veldhuisen B. Alpha-1 antitrypsin Null mutations and severity of emphysema. Respir Med. 2008; 102(6):876-884.

doi: https://doi.org/10.1016/j.rmed.2008.01.009

5. Kohnlein T, Welte T. Alpha-1 antitrypsin deficiency: pathogenesis, clinical presentation, diagnosis, and treatment. Am J Med. 2008; 121(1):3-9.

doi: https://doi.org/10.1016/j.amjmed.2007.07.025

6. Fairbanks KD, Tavill AS. Liver disease in alpha 1-antitrypsin deficiency: a review. Am J Gastroenterol. 2008;103(8):2136-2141; quiz 2142.

doi: https://doi.org/10.1111/j.1572-0241.2008.01955.x

7. Crystal RG. Alpha 1-antitrypsin deficiency, emphysema, and liver disease. Genetic basis and strategies for therapy. J Clin Invest. 1990; 85(5):1343-1352. doi:

https://doi.org/10.1172/JCI114578

8. Barker AF, Siemsen F, Pasley D, D'Silva R, Buist AS. Replacement therapy for hereditary alpha1-antitrypsin deficiency. A program for long-term administration. Chest. 1994;105(5):1406-1410. doi: https://doi.org/10.1378/chest.105.5.1406

9. Abboud RT, Ford GT, Chapman KR. Emphysema in alpha1antitrypsin deficiency: does replacement therapy affect outcome? Treat Respir Med. 2005; 4(1):1-8. doi: https://doi.org/10.2165/00151829-200504010-00001

10. Petrache I, Hajjar J, Campos M. Safety and efficacy of alpha-1antitrypsin augmentation therapy in the treatment of patients with alpha-1-antitrypsin deficiency. Biologics. 2009; 3:193-204. doi: https://doi.org/10.2147/btt.s4414

11. Chapman KR, Burdon JG, Piitulainen E, et al. Intravenous augmentation treatment and lung density in severe alpha1 antitrypsin deficiency (RAPID): a randomised, double-blind, placebo-controlled trial. Lancet. 2015; 386(9991):360-368. doi: https://doi.org/10.1016/S0140-6736(15)60860-1

12. AlphaNet. ADMAP Program: Alpha-1 Disease Management and Prevention Program. 2016; AlphaNet website http://www. alphanet.org/services/ . Accessed October 2016
13. Campos MA, Alazemi S, Zhang G, Wanner A, Sandhaus RA. Effects of a disease management program in individuals with alpha-1 antitrypsin deficiency. COPD. 2009;6(1):31-40. doi: https://doi.org/10.1080/15412550802607410

14. Charlson ME, Pompei P, Ales KL, MacKenzie CR. A new method of classifying prognostic comorbidity in longitudinal studies: development and validation. J Chronic Dis. 1987; 40(5):373-383. doi: https://doi.org/10.1016/0021-9681(87)90171-8

15. Bursac Z, Gauss CH, Williams DK, Hosmer DW. Purposeful selection of variables in logistic regression. Source Code Biol Med. 2008; 3:17. doi: https://doi.org/10.1186/1751-0473-3-17 